\section{Des déterminants cellulaires et viraux contrôlent l'expansion de compartiments intracellulaires séquestrant le VIH-1 dans les macrophages infectés}

Leslie Lepont, Olivier Leymarie, Clarisse Berlioz-Torrent
Université de Paris, Institut Cochin, Inserm, CNRS, laboratoire «Interactions hôte-virus », 27 rue du faubourg Saint Jacques, F-75014

Paris, France.

clarisse.berlioz@inserm.fr

Le facteur de restriction BST2, une barrière cellulaire contre la dissémination des virus enveloppés, et son adversaire, la protéine Vpu du VIH-1

Deux études ont montré que le facteur de restriction ${ }^{1}$ bone marrow stromal antigen 2 (BST2, ou tetherin, ou CD317, ou encore HM1.24) est présent dans les VCC $[6,7]$. La protéine cellulaire BST2/tetherin est I'un des facteurs de restriction du VIH et du virus de l'immunodéficience simienne. Il s'agit d'un acteur majeur de l'immunité innée, restreignant la dissémination de nombreux virus enveloppés. Ce facteur retient physiquement les nouveaux virus formés à la surface de la cellule productrice, réduisant ainsi la production et la dissémination virale $[8,9]$ (Figure 2). Face à cette restriction, les lentivirus ont développé différentes stratégies pour diminuer la quantité de BST2/tetherin présente aux sites de bourgeonnement viral et restaurer ainsi une production virale efficace [10]. La protéine Vpu du VIH-l est l'un des acteurs viraux capables de contrer cette restriction $[8,9]$. Ainsi, de nombreuses études réalisées sur des lignées cellulaires ont montré que Vpu favorise

\footnotetext{
${ }^{1}$ Les facteurs de restriction sont des protéines antivirales cellulaires qui inhibent directement ou indirectement certaines étapes de la multiplication virale. Elles sont parfois produites de façon constitutive, parfois inductibles par l'interféron.
} 


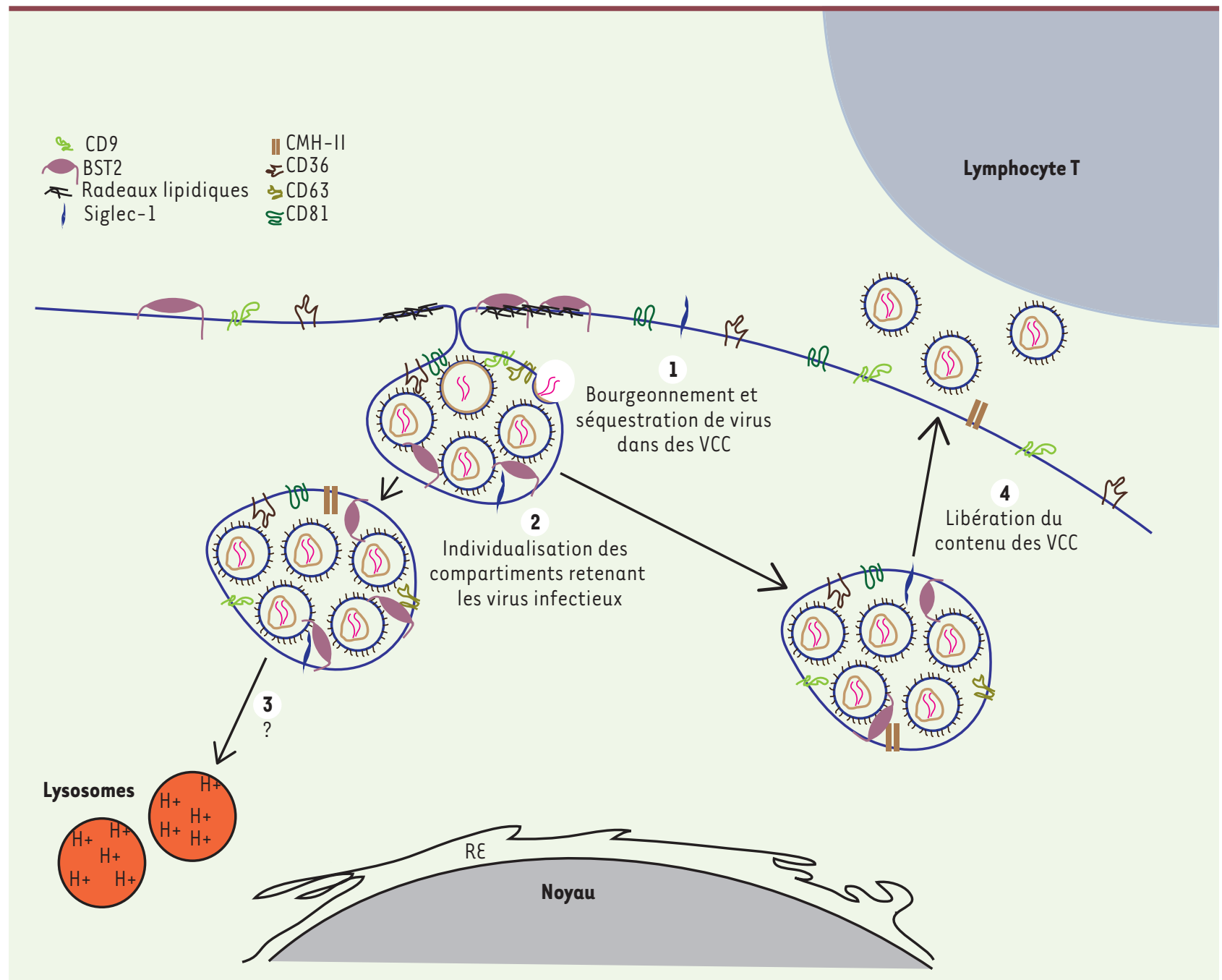

Figure 1. Le VIH-1 s'accumule dans des compartiments intracellulaires connectés à la membrane cellulaire. 1. Lors des étapes tardives du cycle viral, les virions néo-synthétisés s'accumulent dans des compartiments intracellulaires reliés à la membrane, les VCC (virus-containing compartments). Ces VCC sont enrichis en tétraspanines CD9, CD36, CD63 et CD81, mais également en molécules du complexe majeur d'histocompatibilité de classe II (CMH-II), en glycoprotéine Siglec-1 (sialic acid-binding lg-like lectin 1) qui se lie à l'acide sialique, et en protéine BST2 (bone marrow stromal antigen 2). 2. Ces compartiments vont ensuite s'individualiser et perdurer dans la cellule. 3. Le devenir de ces VCC, notamment leur dégradation par les lysosomes, reste mal connu, mais les virions séquestrés peuvent être transférés à un lymphocyte T via la formation de «synapses virologiques » 4 . RE : réticulum endoplasmique.

la libération des particules virales. En absence de $\mathrm{Vpu}$, moins de virions sont relâchés dans le milieu extracellulaire, et des virions s'accumulent dans les cellules productrices (Figure 2). À ce jour, peu d'études décrivent les mécanismes par lesquels Vpu interfère avec la restriction imposée par BST2 dans les macrophages infectés.

Notre étude publiée dans Journal of Virology [11] analyse les contributions respectives du facteur de restriction BST2 et de la protéine virale Vpu dans la production de nouveaux virions, ainsi que la formation et le maintien des VCC séquestrant les virions dans les macrophages infectés. Pour étudier l'impact de Vpu sur la production virale et la formation des VCC dans le macrophage primaire, nous nous sommes appuyés sur un modèle de macrophages dérivés de monocytes isolés à partir de sang humain (monocytederived macrophages, MDM). Les MDM sont infectés avec un virus VIH-l de type «sauvage » (wild-type, WT) ou privé de sa protéine Vpu (Udel). Une quantification de la protéine virale de capside CAp24 associée aux cellules ou présente dans le milieu extracellulaire par la technique ELISA permet une analyse comparative de la production de virions. Ce dosage montre que l'expression de Vpu favorise la libération des particules virales dans le surnageant et réduit la quantité de CAp24 associée à la cellule. Vpu favorise donc la libération des particules virales pro- 


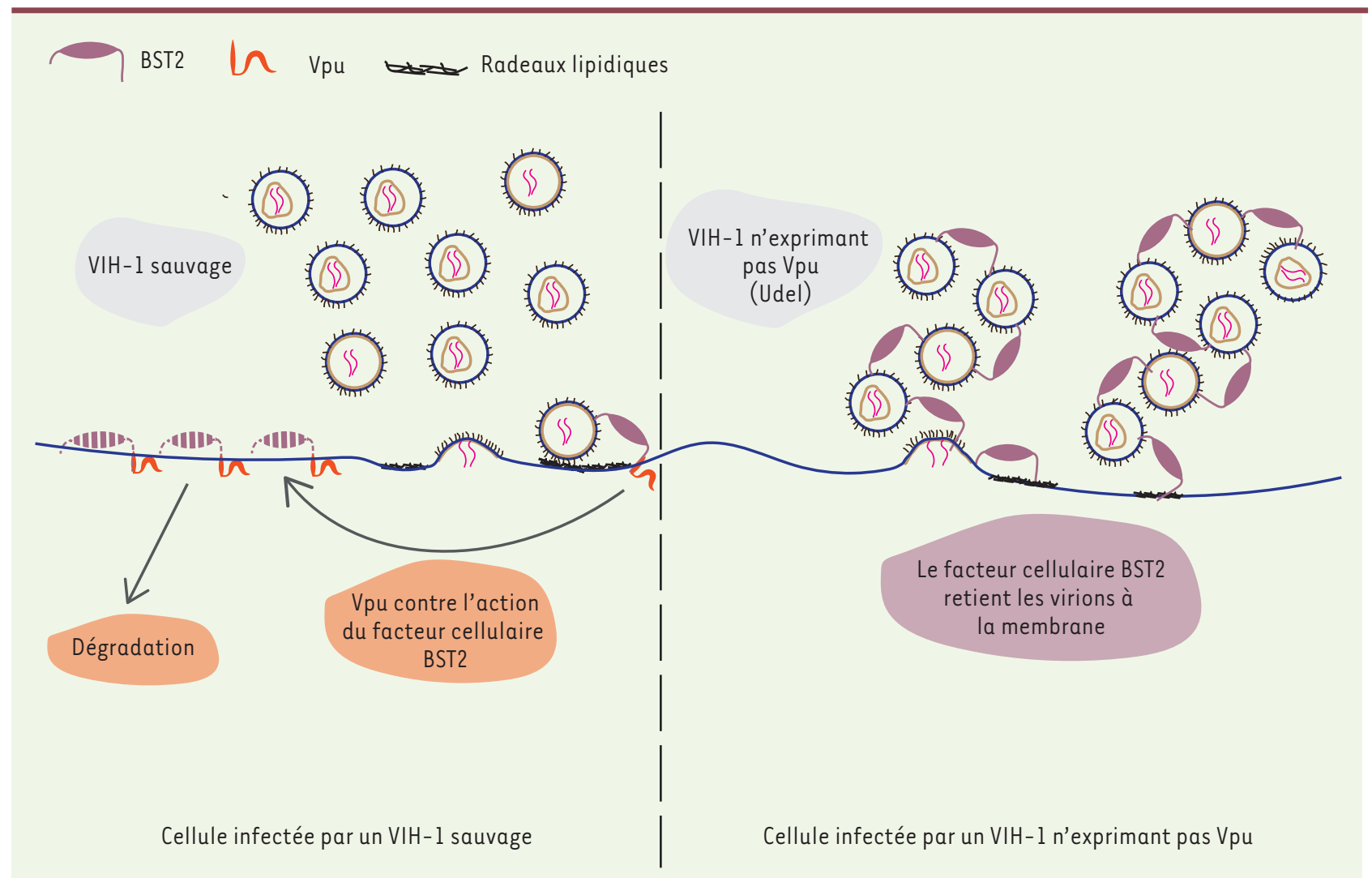

Figure 2. La protéine virale Vpu contrecarre l'action du facteur cellulaire de restriction BST2 et induit sa dégradation. Lors de l'infection par un VIH-l du type « sauvage » (à gauche), les virions néo-formés bourgeonnent à la membrane plasmique et sont relâchés dans le milieu extracellulaire. Lors de l'infection par un VIH-l privé de la protéine Vpu (viral protein U, à droite), les virions néo-formés s'accumulent à la membrane cellulaire. Cette rétention est due à la présence de la protéine BST2 à la surface cellulaire : BST2 s'ancre simultanément dans la membrane plasmique et dans l'enveloppe virale des virions bourgeonnants. Lors de l'infection par un virus de type sauvage, Vpu se lie à BST2 et induit sa dégradation, favorisant la libération des particules virales.

duites par les macrophages. Par ailleurs, lorsque l'expression de BST2 est réduite par transfection des macrophages avec un ARN interférant ciblant l'ARNm de ce gène, le virus VIH-1 Udel (privé de Vpu) est libéré tout aussi efficacement dans le milieu extracellulaire que le virus de type sauvage. L'effet de Vpu sur la production virale est donc lié à l'expression de BST2 dans les macrophages.

\section{La protéine virale Vpu diminue la} quantité globale de protéine BST2 dans la cellule infectée, ainsi que sa présence à la surface des macrophages Nos résultats indiquent que l'expression de Vpu augmente la production virale en contrecarrant le facteur de restriction BST2. Deux activités différentes de Vpu sur BST2 ont été rapportées, principale- ment dans les lignées cellulaires modèles (HEK293T et HeLa, cellules T lymphoblastoïdes (EM). Vpu réduit la quantité globale de protéine BST2 dans les cellules infectées, ainsi que sa présence à la surface de ces cellules en favorisant son adressage aux lysosomes, et limite donc le recyclage de la protéine vers la surface cellulaire. La seconde activité de Vpu réside dans sa capacité à exclure BST2 des sites de bourgeonnement viral et, de ce fait, à limiter son incorporation dans l'enveloppe virale en formation pour permettre la libération des particules virales dans le surnageant [10]. Dans notre étude, l'analyse de la quantité de protéine BST2 présente dans des lysats de MDM infectés, ainsi qu'une analyse de ces cellules par cytométrie en flux, montrent que $V p u$ réduit significativement la quan- tité globale de BST2 dans les MDM et la présence de BST2 à leur surface, un effet dont l'intensité diffère cependant selon les donneurs. L'action de Vpu sur BST2 dans les macrophages est donc semblable à celle décrite auparavant dans différentes lignées cellulaires.

\section{L'intégrité du domaine} transmembranaire et de deux motifs du domaine intracellulaire de Vpu sont nécessaires à son action sur la production des virions

L'action de Vpu sur la production des virions dépend de plusieurs caractéristiques moléculaires de cette protéine. Une interaction directe entre le domaine transmembranaire de Vpu et celui de BST2 est essentielle à son action sur la présence de BST2 à la surface des cellules infectées 
[10]. Par ailleurs, deux motifs présents dans la séquence protéique de Vpu participent à l'exclusion de BST2 du site de bourgeonnement viral et à la diminution de l'expression de surface de BST2 : (1) un motif de type dileucine, $\varepsilon_{59} V_{S A L}{ }_{63} V$, présent dans la seconde hélice $\alpha$ du domaine cytoplasmique de Vpu, et (2) deux sérines phosphorylables $\left(S_{52}\right.$ et $S_{56}$ ), requises pour son interaction avec l'\&3 ubiquitine ligase $\beta$-TRCP [10]. Nous avons donc construit, par mutagénèse dirigée, différents clones proviraux de $\mathrm{VIH}-1$ porteurs de mutations dans la séquence codant Vpu, et particulièrement dans celle codant son domaine transmembranaire ou l'un des deux motifs du domaine intracellulaire sus-mentionnés. L'évaluation de la production virale pour ces différents mutants montre que l'intégrité du domaine transmembranaire de Vpu est critique pour obtenir une production virale optimale dans les macrophages. En outre, il apparaît que les deux motifs du domaine cytoplasmique de Vpu contribuent, dans une moindre mesure, à l'action de Vpu sur la dissémination virale. Deux équipes ont rapporté un enrichissement de protéines BST2 dans les VCC lors de l'infection par le VIH-1 $[6,7]$. Nous avons donc analysé la distribution de BST2 dans ces compartiments par microscopie confocale, et défini l'impact de l'expression de Vpu sur cette distribution. Nous observons qu'en absence de Vpu, BST2 est localisée dans les VCC contenant les virons VIH-l, alors que la protéine n'est plus associée à ces compartiments lorsque les cellules infectées expriment Vpu. Par ailleurs, à l'aide du logiciel de reconstitution 3D IMARIS, nous avons montré que la présence de Vpu réduit le volume de ces structures. En absence de BST2, les VCC sont toujours présents, mais l'effet de Vpu sur leur volume n'est plus visible. La présence de BST2 favorise donc la formation de grands VCC, mais n'est pas indispensable à la formation de ces compartiments. Enfin, nous avons analysé le volume des VCC dans des macrophages infectés par les virus mutés dans le domaine transmembranaire, le motif de type dileucine, ou les deux sérines phosphorylables de Vpu. L'analyse en microscopie confocale a révélé un rôle plus complexe de Vpu sur la taille de ces compartiments. En effet, nos résultats suggèrent que Vpu contrôle la taille de ces compartiments en excluant BST2 des VCC via l'interaction directe entre les domaines transmembranaires des deux protéines, et en agissant sur le trafic intracellulaire de BST2. Ils indiquent également que d'autres facteurs cellulaires participeraient à la formation et au contrôle du volume des VCC.

\section{Perspectives}

Notre étude apporte de nouvelles informations sur le rôle de la protéine virale Vpu et du facteur de restriction BST2 dans la séquestration intracellulaire des virions néo-synthétisés dans les macrophages infectés par VIH-l. Nous montrons que le volume des compartiments intracellulaires abritant les virions est contrôlé par Vpu. Cette activité de Vpu requiert l'intégrité de son domaine transmembranaire, ainsi que celle d'un motif de type dileucine et de deux sérines phosphorylées de son domaine cytosolique. Ce processus dépend de la capacité de Vpu à diminuer la présence de BST2 au site de bourgeonnement viral. Vpu réduit ainsi la quantité de virions accumulés au site de bourgeonnement et séquestrés ultérieurement dans les VCC. Ainsi, l'expression de Vpu limite l'expansion de ces compartiments. Par ailleurs, notre étude souligne qu'à travers d'autres activités non identifiées, Vpu limite l'enrichissement de BST2 dans les VCC.

La présence de ces compartiments intracellulaires confère donc aux macrophages un rôle de sanctuaire pour le virus VIH-1. Ce réservoir viral, sousestimé pendant de nombreuses années, est aujourd'hui au cœur de nombreuses recherches. Ainsi, une étude récente montre que des macrophages présents dans les tissus de l'urètre de patients sous traitement antirétroviral depuis plusieurs années possèdent des compartiments intracellulaires contenant des virus réplicatifs qui sont transmissibles à des cellules cibles après réactivation [12]. L'étude des mécanismes contrôlant la formation et la persistance de ces compartiments dans les macrophages est donc un enjeu majeur pour progresser dans l'éradication de ces sanctuaires viraux. $\diamond$

Cellular and viral determinants control HIV- 1 virus-containing compartment expansion in infected macrophages

\section{REMERCIEMENTS}

Le laboratoire est soutenu par l'ANRS et SIDACTION.

\section{LIENS D'INTÉRÊT}

Les auteurs déclarent n'avoir aucun lien d'intérêt concernant les données publiées dans cet article.

\section{RÉFÉRENCES}

1. Tan J, Sattentau QJ. The HIV-1-containing macrophage compartment: a perfect cellular niche? Trends Microbiol 2013 ; 21 : 405-12.

2. Orenstein JM, Meltzer MS, Phipps T, et al. Cytoplasmic assembly and accumulation of human immunodeficiency virus types 1 and 2 in recombinant human colony-stimulating factor-1-treated human monocytes: an ultrastructural study. J Virol 1988; 62 : 2578-86.

3. Jouve M, Sol-Foulon N, Watson S, et al. HIV-1 buds and accumulates in nonacidic endosomes of macrophages. Cell Host Microbe 2007 ; 2 : 85-95.

4. Deneka M, Pelchen-Matthews A, Byland R, et al. In macrophages, HIV-l assembles into an intracellular plasma membrane domain containing the tetraspanins CD81, CD9, and CD53. J Cell Biol 2007 ; $177: 329-41$

5. Pelchen-Matthews A, Kramer B, Marsh M. Infectious HIV-l assembles in late endosomes in primary macrophages. J Cell Biol 2003 ; 162 : 443-55.

6. Chu H, Wang JJ, Qi M, et al. Tetherin/BST-2 is essential for the formation of the intracellular virus-containing compartment in HIV-infected macrophages. Cell Host Microbe $2012 ; 12: 360-72$.

7. Giese S, Marsh M. Tetherin can restrict cell-free and cell-cell transmission of HIV from primary macrophages to T cells. PLoS Pathog $2014 ; 10$ : e1004189.

8. Neil SJD, Zang T, Bieniasz PD. Tetherin inhibits retrovirus release and is antagonized by HIV- $1 \mathrm{Vpu}$. Nature 2008 ; $451:$ : 425-30.

9. Damme N Van, Goff D, Katsura C, et al. The interferoninduced protein BST-2 restricts HIV-1 release and is downregulated from the cell surface by the viral Vpu protein. Cell Host Microbe 2008 ; 3 : 245-52.

10. Roy N, Pacini G, Berlioz-Torrent C, et al. Mechanisms underlying HIV-1 Vpu-mediated viral egress. Front Microbiol $2014 ; 5: 177$.

11. Leymarie 0 , Lepont L, Versapuech M, et al. Contribution of the cytoplasmic determinants of Vpu to the expansion of virus-containing compartments in HIV-1-infected macrophages. J Virol 2019 ; 93 : e00020-19.

12. Ganor Y, Real F, Sennepin A, et al. HIV-I reservoirs in urethral macrophages of patients under suppressive antiretroviral therapy. Nat Microbiol 2019 ; 4 : 633-44. 